Graham gave himself 5 minims of a 5 per cent. solution rendered alkaline by the addition of 3 per cent. sod. carbonate without bad result. The urine 15 minutes after showed slight fluorescence. After an hour the urine showed very distinct fluorescence, which increased markedly for four days after it was passed, i.e., with greater alkalinity To prove that the tissues were rendered fluorescent he injected under the shaved skin of a guinea-pig 5 minims of a 5 per cent. solution. He then removed 3 square inches of skin with the subcutaneous and muscular tissue, and stretched these over a ring. He then passed the light from a Finsen-Reyn lamp through, and found that the whole of the skin and the subcutaneous tissue were very fluorescent, being 1 mil. thick, but in the muscle 3 mil. thick the fluorescence was not so evident. Only a small dose is necessary - 1-5 min. of 5 per cent. solution-and it should be used within two days of being made. The reaction after light is usually over by the third day; after Asculin it lasts four to seven days longer, and subsequent exposures continue to produce greater reactions for four to five days afterwards. No ill effects have followed 100 injections. The chief value Graham thinks to be in isolated, obstinate tubercular nodules, remaining after a preliminary course of Finsen treatment. It also softens thickened fibrous scars left after scraping for lupus, which render nodules often difficult of access.

1 Annals of Surgery. Dec., 1905. 2 Berlin Klin. Woch, No. 44 A, 1905, Brit. Med. Jour., Jan. 6, 1906. ${ }^{3}$ Edin. Jour., Jan., 1906. ¿ Brit. Med. Jour., Feb. 3, 1906. 5 Med. Rec., Nov. 18, 1905. - Archives of Röntgen Ray, Mar., 1906. 7 Annals of Surgery, Dec., 1905. "Archives of Röntgen Ray, Feb., 1905. - Lancet, Deo. 16, 1905.

\section{DISEASES OF THE BLOOD.}

Arterio-sclerosis.-Among the causes which lead to this condition are the infective processes, such as typhoid, septicæmia, syphilis, and lead-poisoning. Barr, Christophers, and others think that the bacillus coli has a similar power, and show that the blood of persons who suffer from the disease much more often agglutinates the bacillus coli than the blood of normal persons does. In cases examined by John Hay, the percentage of persons thus affected whose blood caused clumping was 53, as compared to 20 in normal individuals. Alcohol does not seem to have much direct effect, but Barr ${ }^{1}$ claims that continued high tension and an excess of nitrogenous food are powerful factors. In connection with this point he accepts the recent results of Chittenden as to the small amount actually needed, but it has been pointed out elsewhere that these results need confirmation, and that it is totally unknown how individuals on such a restricted diet stand a sudden attack of disease or even very heavy labour. It is, indeed, noticeable that such persons find a great difficulty in increasing their food if it is desirable to do so. Barr, indeed, confesses that a great reduction of the purin bodies in food may sometimes be harmful, for, as Oliver has pointed out, these substances act during digestion as distributors of lymph to the tissues, an office which the nutritious elements of food themselves are unable to carry out. Still, in the treatment of arterio-sclerosis Barr thinks that a low proteid diet is valuable and milk is in itself somewhat objectionable; as to drugs he recommends thyroid extract, the iodides and benzoates, and saline purgatives. On the other hand, M. Fränkel ${ }^{2}$ thinks that iodine is useless in this disease, but finds considerable benefit from Trunecek's serum: When given hypodermically the process is tedious and painful, but antisclerosin, or the dried salts contained in it, can equally well be given by the mouth. The remedy may do good even in advanced cases, but it is still more valuable when given as a prophylactic as soon as we notice a high-tension pulse, slight dyspnœa, præcordial oppression, visual troubles, or the early signs of gout.

Leucocytosis after Digestion. - It appears that the absence of a normal increase of white cells after digestion is a strong, though not absolute, indication of gastric or hepatic cancer. The difficulties of diagnosing this condition are so great that any additional evidence is valuable. Goodall and others made observations on dogs and found the maximum rise four hours after food. The increase was chiefly in the lymphocytes and sometimes was shown also in the polymorphonuclear cells. It was also proved that the increase of cells was not due to increased action in the intestinal walls, but chiefly, if not entirely, to the bone-marrow. Other writers find that the maximum rise occurs in human beings two hours after food and is generally absent in gastric and hepatic cancer. In cases of gastric disease other than cancer, in tuberculosis, nephritis, diabetes, and other diseases, it is present except in the terminal stage, when gastric functions are completely abolished. Thus in ordinary cases its absence is strongly presumptive of malignant disease.

${ }^{1}$ Brit. Med. Jour., Jan. 20. ' Amer. Jour. Med. Sci., Jan.

\section{CARCINOMA.}

(Continued from page 124.)

On the Treatment of Tuberculosis of the Bones and Joints. - In the discussion ${ }^{4}$ on the treatment of tubercular joints at the recent International Surgical Congress there was a surprising advocacy of conservative measures. All are agreed on two principles-namely, those of rest and of hygienic treatment as having the greatest tendency to promote recovery. Tubercular disease is one in which natural healing can and will take place if only the general and local conditions are favourable. Kocher was the only speaker who expressed himself in favour of early operation in all cases. But in addition to immobilisation of the joint and good surroundings for the patient there are three local measures strongly advocated by various authorities as hastening the process of cure. Bier obtains the best results from his method of passive congestion induced by means of a rubber band applied on the proximal side of the joint. The band is applied for one to three hours a day, and should not be tight enough to cause pain. The method is employed even in the presence of sinuses, the dressings of which are removed whilst the treatment is 